\title{
Virtual screening approach to identifying influenza virus neuraminidase inhibitors using molecular docking combined with machine-learning-based scoring function
}

\author{
Li Zhang ${ }^{1,2, *}$, Hai-Xin $\mathrm{Ai}^{1,2,3, *}$, Shi-Meng $\mathrm{Li}^{1}$, Meng-Yuan $\mathbf{Q i}^{1}$, Jian Zhao ${ }^{1}$, Qi Zhao ${ }^{4}$ \\ and Hong-Sheng Liu ${ }^{1,2,3}$ \\ ${ }^{1}$ School of Life Science, Liaoning University, Shenyang 110036, China \\ ${ }^{2}$ Research Center for Computer Simulating and Information Processing of Bio-macromolecules of Liaoning Province, \\ Shenyang 110036, China \\ ${ }^{3}$ Engineering Laboratory for Molecular Simulation and Designing of Drug Molecules of Liaoning, Shenyang 110036, China \\ ${ }^{4}$ School of Mathematics, Liaoning University, Shenyang 110036, China \\ *The first two authors should be regarded as joint first authors \\ Correspondence to: Hong-Sheng Liv, email: liuhongsheng@Inu.edu.cn
}

Keywords: influenza virus, neuraminidase inhibitor, virtual screening, machine learning, scoring function

Received: August 02, $2017 \quad$ Accepted: August 28, $2017 \quad$ Published: September 15, 2017

Copyright: Zhang et al. This is an open-access article distributed under the terms of the Creative Commons Attribution License 3.0 (CC BY 3.0), which permits unrestricted use, distribution, and reproduction in any medium, provided the original author and source are credited.

\section{ABSTRACT}

In recent years, an epidemic of the highly pathogenic avian influenza H7N9 virus has persisted in China, with a high mortality rate. To develop novel anti-influenza therapies, we have constructed a machine-learning-based scoring function (RFNA-Score) for the effective virtual screening of lead compounds targeting the viral neuraminidase (NA) protein. RF-NA-Score is more accurate than RF-Score, with a root-mean-square error of 1.46, Pearson's correlation coefficient of 0.707 , and Spearman's rank correlation coefficient of 0.707 in a 5-fold cross-validation study. The performance of RF-NA-Score in a docking-based virtual screening of NA inhibitors was evaluated with a dataset containing 281 NA inhibitors and 322 noninhibitors. Compared with other docking-rescoring virtual screening strategies, rescoring with RF-NA-Score significantly improved the efficiency of virtual screening, and a strategy that averaged the scores given by RF-NA-Score, based on the binding conformations predicted with AutoDock, AutoDock Vina, and LeDock, was shown to be the best strategy. This strategy was then applied to the virtual screening of NA inhibitors in the SPECS database. The 100 selected compounds were tested in an in vitro H7N9 NA inhibition assay, and two compounds with novel scaffolds showed moderate inhibitory activities. These results indicate that RF-NA-Score improves the efficiency of virtual screening for NA inhibitors, and can be used successfully to identify new NA inhibitor scaffolds. Scoring functions specific for other drug targets could also be established with the same method.

\section{INTRODUCTION}

Since the first report of human infection with the avian influenza H7N9 virus in China in March 2013, this new viral subtype has caused sustained annual epidemic in subsequent years $[1,2]$. The newly issued monthly
World Health Organization (WHO) risk assessment summary at the human-animal interface [3] has reported that, as of June 2017, avian influenza H7N9 has caused 1533 laboratory-confirmed human infections, including at least 592 deaths. Recent studies have shown that the pandemic potential of avian influenza H7N9 is great [4, 
5]. The emergence of new types of influenza viruses and the spread of drug-resistant strains [6] make the influenza virus a serious public-health threat.

Neuraminidase (NA) is an important surface glycoprotein of the influenza viruses. The main role of NA is to remove the sialic acid groups from glycoproteins at the surfaces of host cells, resulting in the release of virion progeny from infected cells. NA also allows the virus to move through the mucus layer of the respiratory tract in the early stage of infection, which is important if the virus is to reach and infect epithelial cells $[7,8]$. NA also prevents the formation of viral aggregates at the surfaces of host cells, enhancing viral infectivity [9]. The amino acid residues in the active site of NA are highly conserved among all natural strains of influenza virus infections [10]. These features of NA make it an attractive target for the control and treatment of influenza virus [11]. Several NA inhibitors that target the active site of NA have been developed and shown to be effective in clinical trials [12-15]. Two NA inhibitors, oseltamivir (Tamiflu®) and zanamivir (Relenza ${ }^{\circledR}$ ), have been approved by the Food and Drug Administration (FDA) in the USA for the treatment and prevention of influenza viral infection. However, the emergence and wide-ranging spread of oseltamivir-resistant strains $[6,16]$, the limited use of zanamivir because its oral bioavailability is poor [17], and the emergence of new and more aggressive strains, such as avian H5N1 and H7N9 [1], have amplified the need for the development of new and more-effective antiviral compounds.

Virtual screening based on molecular docking is becoming a powerful tool in identifying lead compounds $[18,19]$, and a series of potential NA inhibitors has been identified with this method [20-22]. Current NA inhibitor virtual screening studies usually use the score (binding affinity value) given by scoring functions integrated into molecular docking software to rank the compounds. Therefore, this score is the main basis for the selection of potential inhibitors. These scoring functions are designed to recognize correct binding conformations in the docking process, but perform poorly in ranking the compounds correctly in the order of their activity against a target. Cheng et al. examined the abilities of 16 popular scoring functions to predict binding affinities using a dataset containing 195 protein-ligand complexes and their experimental binding affinities, and showed that the best scoring function achieved a Pearson's correlation coefficient of 0.644 [23]. The low accuracy of the scoring function is a major factor limiting the efficiency of molecular-docking-based virtual screening [24].

In recent years, machine-learning methods have been successfully used in many aspects of pharmaceutical research, such as drug-target interactions prediction $[25,26]$, synergistic drug combinations prediction [27, 28], and drug toxicity prediction [29]. In the scope of virtual screening, new scoring functions based on modern machine-learning regression models have been introduced, and have been shown to outperform a wide range of classical scoring functions [30]. Khamis et al. [31] comprehensively evaluated 12 machine-learningbased scoring functions and 20 classical scoring functions. Their results showed that the machine-learning-based scoring functions were a substantial improvement on classical scoring functions in both scoring power (binding affinity prediction) and ranking power (relative ranking prediction). Ashtawy and Mahatrapa [32] reported that machine-learning scoring functions using the random forests algorithm or boosted regression trees were most frequently associated with the best performance. With the accumulation of protein-ligand complex structural data in public databases, it is possible to design scoring functions for the more-efficient prediction of the binding affinity of specific proteins or protein families.

In this study, we developed an NA-specific scoring function using the random forests algorithm for the efficient virtual screening of NA inhibitors. The efficiency of various virtual screening strategies combining different docking software and scoring functions was then evaluated on a test dataset containing 281 NA inhibitors and 322 noninhibitors. The best strategy was used to virtually screen for NA inhibitors in the SPECS database.

\section{RESULTS}

\section{Performance of RF-NA-Score}

Using 36 RF-Score features, 11 Vina features, and the experimental binding affinity values of the training set complexes (67 NA-ligand complexes from the PDBbind database version 2016) as input data, a scoring function specific for Influenza A virus NA, designated RF-NAScore, was trained with the method proposed by Ballester and Mitchell [33-35]. The performance of RF-NA-Score was rigorously validated with 5-fold cross-validation (5-CV) and leave-one-out cross-validation (LOOCV) methods. The performance measures are presented in Table 1. For comparison, RF-Score was also retrained on the refined set of the latest version of the PDBbind database (version 2016), which contains more complexes and should result in a more robust scoring function. The performance of RF-Score in predicting the binding affinities of the 67 NA-ligand complexes is also shown in Table 1.

As shown in Table 1, RF-NA-Score achieved rootmean-square errors (RMSEs) of 1.46, 1.48, and 1.50 on $5-\mathrm{CV}, \mathrm{LOOCV}$, and resampling test, respectively, which are obviously smaller than the value of 1.63 achieved with RF-Score, indicating that the difference between the measured binding affinities and those predicted by RFNA-Score was smaller than that of RF-Score. On two other performance measures, Pearson's correlation coefficient (Rp) and Spearman's rank correlation coefficient (Rs), 
Table 1: Performance measures of RF-NA-Score and RF-Score for 67 NA-ligand complexes, measured with the root-mean-square error (RMSE), Pearson's correlation coefficient (Rp), and Spearman's rank correlation coefficient (Rs) for the predicted and measured binding affinities

\begin{tabular}{lcccc}
\hline Method & $\begin{array}{c}\text { Number of test } \\
\text { complexes }\end{array}$ & RMSE & Rp & Rs \\
\hline 5-CV fold1 & 13 & 1.47 & 0.846 & 0.841 \\
5-CV fold2 & 13 & 1.86 & 0.740 & 0.714 \\
5-CV fold3 & 13 & 1.51 & 0.630 & 0.758 \\
5-CV fold4 & 14 & 1.57 & 0.577 & 0.565 \\
5-CV fold5 & 14 & 0.88 & 0.744 & 0.657 \\
5-CV average & 13 & 1.46 & 0.707 & 0.707 \\
70\% resampling & 20 & 1.50 & 0.712 & 0.717 \\
average & 67 & 1.48 & 0.722 & 0.711 \\
LOOCV & 67 & 1.63 & 0.670 & 0.593 \\
RF-NA-Score & & & & \\
\hline
\end{tabular}

RF-NA-Score was also better than RF-Score. This means that RF-NA-Score not only produces a higher linear correlation between the predicted and measured binding affinities, but also predicts a more accurate binding affinity rank for the ligands. These features should make virtual screening more efficient. The performance measures for RF-NA-Score and RF-Score suggest that RF-NA-Score is more accurate in predicting the binding affinities between ligands and NA, and could therefore be more efficient in the virtual screening of NA inhibitors.

\section{Validation of the accuracy of molecular docking software}

Because the accurate prediction of the complex structures combining NA and ligands is important for the use of scoring functions such as RF-NA-Score in virtual screening, we validated the accuracy of AutoDock, AutoDock Vina, and LeDock by comparing the predicted and experimentally determined complex structures of four NA inhibitors (oseltamivir carboxylate, zanamivir, laninamivir, and peramivir). Figure 1 shows the structures of NA complexed with oseltamivir carboxylate (Figure 1A), zanamivir (Figure 1B), laninamivir (Figure 1C), and peramivir (Figure 1D) predicted by AutoDock (cyan), AutoDock Vina (magenta), and LeDock (yellow), superimposed on their corresponding crystal structures (green). Table 2 shows the root-mean-square deviation (RMSD) between the predicted and experimentally determined binding conformations of the four inhibitors. It can be seen from Figure 1 that all the predicted binding conformations are very close to the crystal structures. The RMSD values (Table 2) are very low, with an average of around $0.76 \AA$ and no values $>2 \AA$, especially for AutoDock Vina and LeDock, in which the RMSD values are all $<1 \AA$. These results indicate that all three molecular docking software tools are accurate in docking ligands to influenza NA.

\section{Evaluation of RF-NA-Score in the virtual screening of NA inhibitors}

With a comprehensive search of the literature and the bindingDB database (see details in Materials and Methods), we collected a ligand dataset containing 281 inhibitors and 322 noninhibitors of the group 2 NA enzymes of the influenza viruses. With this dataset, RFNA-Score was evaluated for its effectiveness in virtually screening for influenza virus NA inhibitors using the strategy illustrated in Figure 1. Briefly, these ligands were docked to the crystal structure of H7N9 NA (PDB ID: 4MWJ) using the three docking software tools AutoDock, AutoDock Vina, and LeDock. The top-scoring binding complexes predicted by each software were then rescored with RF-Score and RF-NA-Score. The performances of the virtual screening strategies using the different docking software tools combined with different scoring methods were evaluated by comparing their score distributions of inhibitors and noninhibitors and their receiver operating characteristic (ROC) curves.

The score distributions of the inhibitors and noninhibitors are shown in Figure 2. Figure 2A-2C show the distributions of the original scores of the docking software tools. Although the peak values for the distributions of the inhibitors are greater than those of the noninhibitors for all three software tools, the distributions are strongly overlapping, which makes it difficult to distinguish inhibitors from noninhibitors. Rescoring with RF-Score increased the separation of the peak values and reduced the overlap (Figure 2E-2G), indicating that the 
Table 2: RMSD (in $\AA$ ) between the predicted and experimentally determined binding conformations of four inhibitors

\begin{tabular}{lcccc}
\hline Software & Oseltamivir carboxylate & Zanamivir & Laninamivir & Peramivir \\
\hline AutoDock & 1.43 & 1.55 & 1.23 & 0.58 \\
AutoDock Vina & 0.41 & 0.61 & 0.65 & 0.43 \\
LeDock & 0.35 & 0.69 & 0.69 & 0.50 \\
\hline
\end{tabular}

effectiveness of virtual screening was improved by RFScore. However, in the region with high scores (e.g., RFScore $>7$ ), the inhibitors and noninhibitors still seriously overlapped. This can be quantified by the enrichment factor at $10 \%$ (EF10) which reflects the power of the virtual screening method in the enrichment of inhibitors in top $10 \%$ of results. As shown in Figure 2E-2G, the values of EF10 are decreased (except LeDock) after rescoring with RF-Score. Figure 2I-2K show the distribution of the scores provided by RF-NA-Score. RF-NA-Score clearly outperforms RF-Score and the original scores. The separation of the peak values is significantly improved and the overlap between the inhibitors and noninhibitors is clearly smaller than those resulting from RF-Score and the original scores. There are also significantly more inhibitors in the region of high scores. The values of EF 10 are significantly improved. Furthermore, the EF10 value of Vina (2.11) is close to the upper bound of 2.15. These results illustrate the effectiveness of RF-NA-Score in virtual screening.

We also computed an average score for each scoring method by averaging the scores of the three complex structures predicted with the different docking software tools. The distributions of the average scores are shown in Figure 2D, 2H, and 2L. Averaging the scores reduced the overlap of score distributions of inhibitors and noninhibitors for all three scoring methods. And the average RF-NA-Score displayed the best performance.

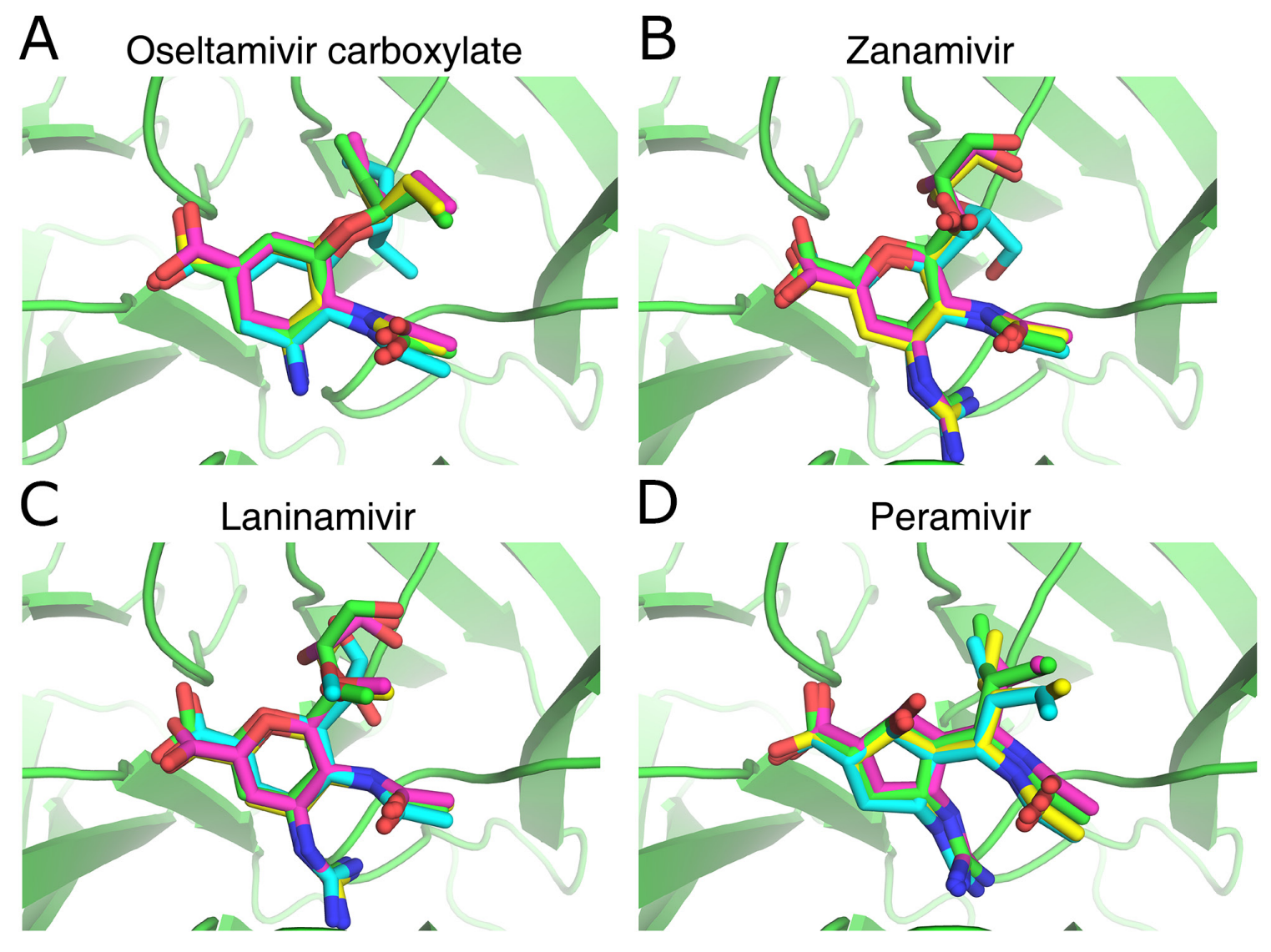

Figure 1: Comparison of the NA inhibitor binding conformations predicted with the docking softwaretools and their corresponding crystal structures. (A) Oseltamivir carboxylate, PDB ID: 4MWQ; (B) zanamivir, PDB ID: 4MWR; (C) laninamivir, PDB ID: 4MWU; (D) peramivir, PDB ID: 4MWV. Small molecules with green, cyan, magenta, and yellow skeletons were derived from crystal structures and structures predicted with AutoDock, AutoDock Vina, and LeDock, respectively. The figure was prepared with PyMOL. 
The EF10 value of average RF-NA-Score is 2.04 which is higher than that of the other two average method (1.82 and $1.25)$ and slightly lower than that of vina combined with RF-NA-Score (2.11).

Student's $t$ test was used to evaluate the significance of the differences between the mean scores for the inhibitors and noninhibitors. The $\mathrm{p}$ value for the average RF-NA-Score strategy was $2.04 \times 10^{-52}$, which was the lowest $\mathrm{p}$ value obtained for all strategies, and clearly suggests that the average RF-NA-Score outperformed the other strategies.

The ROC curves and the areas under the ROC curves (AUCs) are presented in Figure 3. The ROC curve analysis is a well-recognized method of evaluating how good a model is at selecting known active molecules and discarding inactive molecules $[36,37]$. The AUC values range from 0.5 (corresponding to a random model) to 1 (corresponding to an ideal model). In general, the greater the AUC, the more effective the virtual screening strategy is in discriminating active from inactive compounds. Comparing the AUC values of the different strategies clearly showed that RF-NA-Score outperformed the original score and RF-Score when combined with any of the three docking software tools. Figure 3 demonstrates that the best strategy is the average RF-NA-Score, which achieved an AUC value of 0.837. Overall, the results obtained from the ROC curve analysis are consistent with those obtained by comparing the scores distributions.

These results suggest that rescoring with RF-NAScore significantly improves the efficiency of virtual screening for influenza virus NA inhibitors. Among these virtual screening strategies, the best strategy involved docking with AutoDock, AutoDock Vina, or LeDock, rescoring with RF-NA-Score, and then averaging the scores. This strategy was used in subsequent virtual screening.

\section{Screening the SPECS database}

The best virtual screening strategy was used to screen candidate inhibitors of NA in a compound library containing 52,631 lead-like compounds $(250<$ molecular
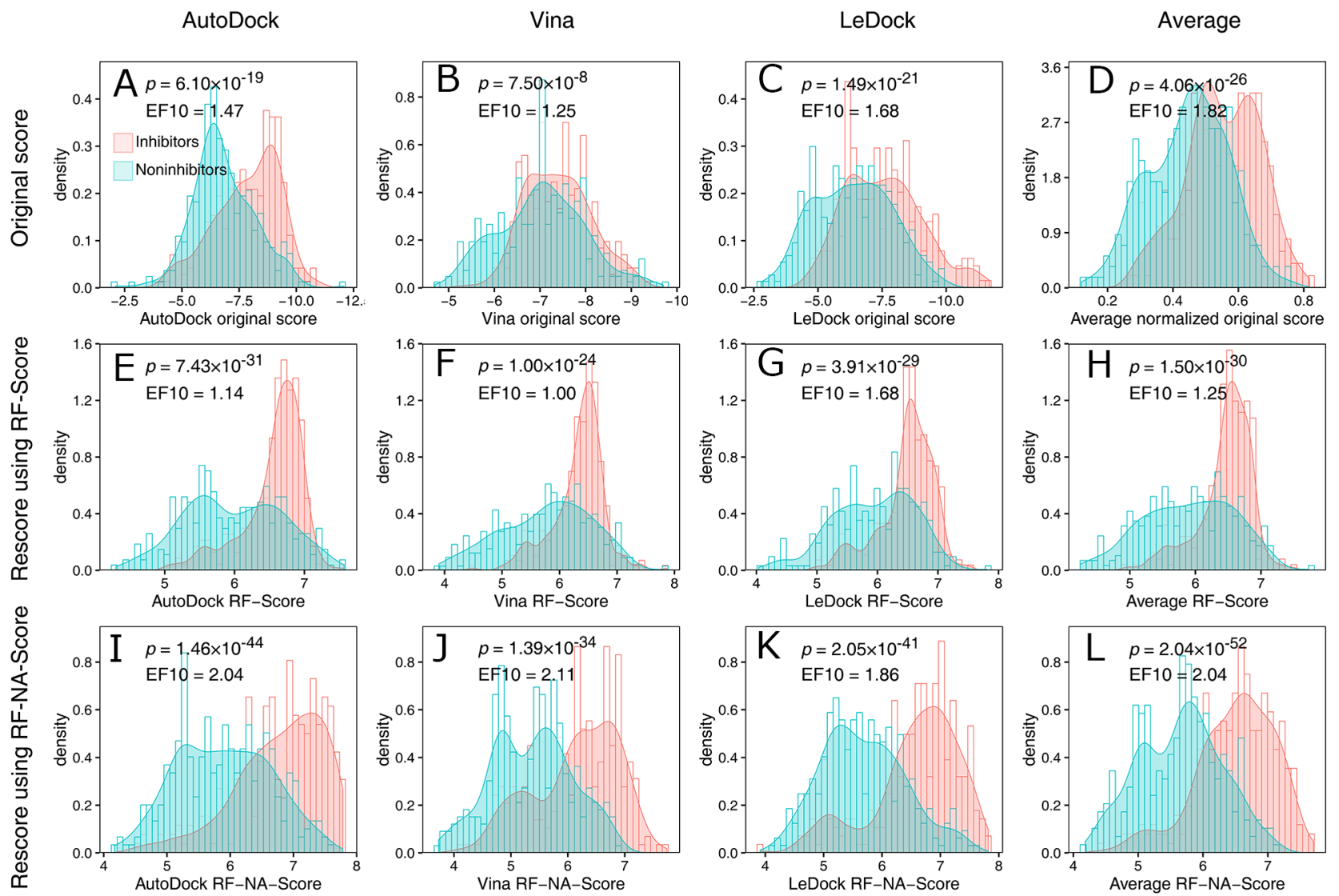

Figure 2: Score distributions of inhibitors (red) and noninhibitors (blue) provided by the docking software (A, B, C, D), RFScore (E, F, G, H), and RF-NA-Score (I, J, K, L) based on the top-scoring docking results of AutoDock (A, E, I), AutoDock Vina (B, F, J), and LeDock (C, $\mathrm{G}, \mathrm{K})$, and the average scores of these three software tools ( $\mathrm{D}, \mathrm{H}, \mathrm{L})$. To average the original scores, the scores were normalized to a range of $0-1$ and then the average was calculated, because different scoring ranges are given by different docking software tools. The $\mathrm{p}$ values were calculated with Student's t test. EF10 is the enrichment factor at 10\%. The upper bound of EF10 is 2.15. 
weight $<350$, and $\log \mathrm{P}<3.5$ ) in the SPECS database. After virtual screening, the 1000 compounds with the best average RF-NA-Score scores were clustered, and 100 compounds with wide chemical diversity were selected as candidate inhibitors.

These compounds were tested in an in vitro H7N9 NA inhibition assay, using oseltamivir carboxylate as the positive control. Two of the compounds, AH034/11365875 and AH-262/08373040, were found to be active at concentrations of $100 \mu \mathrm{M}$ (Figure 4), with inhibition rates of $40.8 \%$ and $31.0 \%$, respectively. The dose-response effects of these two compounds were then evaluated to determine the half-maximal inhibitory concentrations $\left(\mathrm{IC}_{50}\right)$, which were $107.0 \mu \mathrm{M}$ and 194.2 $\mu \mathrm{M}$, respectively (Figure 5). These results suggest that AH-034/11365875 and AH-262/08373040 are moderate NA inhibitors.

The novelty of these two new NA inhibitors was also analyzed by calculating their structural similarity to the 281 previously reported inhibitors. The pairwise similarity of these inhibitors was calculated with the Tanimoto coefficient based on atom pair descriptors [38, 39]. The similarity of compounds AH-034/11365875 and AH-262/08373040 to the most similar compounds among the previously reported inhibitors was 0.281 and 0.373 , respectively. Thus, these two compounds have very low similarity to previously reported inhibitors, suggesting that $\mathrm{AH}-034 / 11365875$ and $\mathrm{AH}-262 / 08373040$ are

B
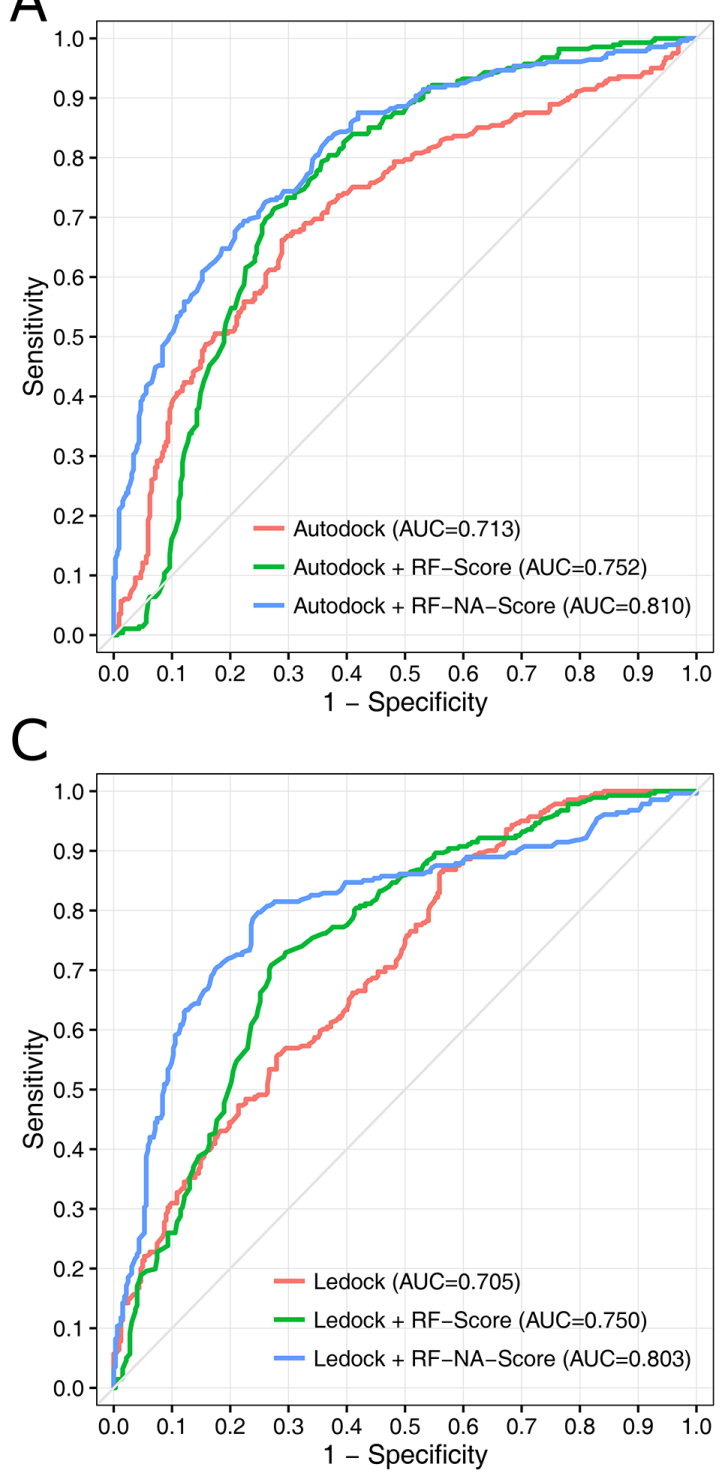

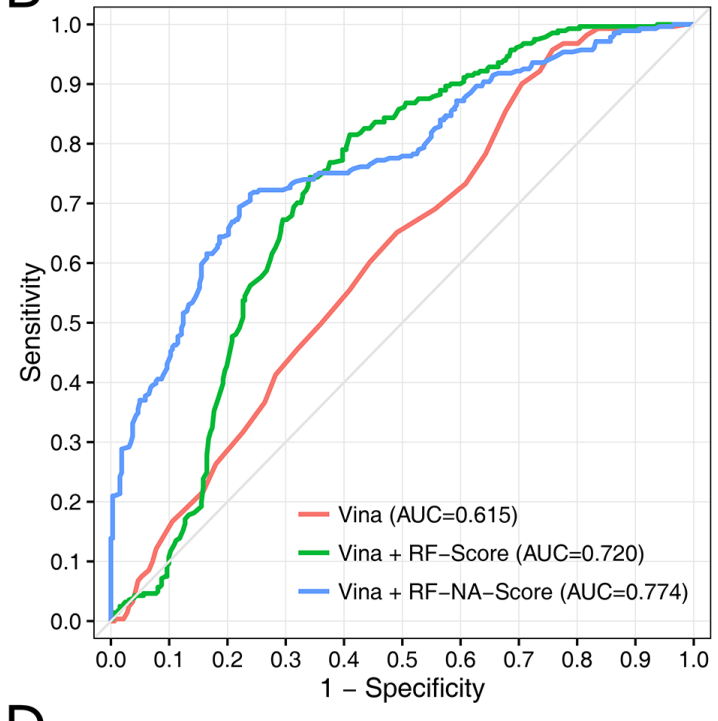

D

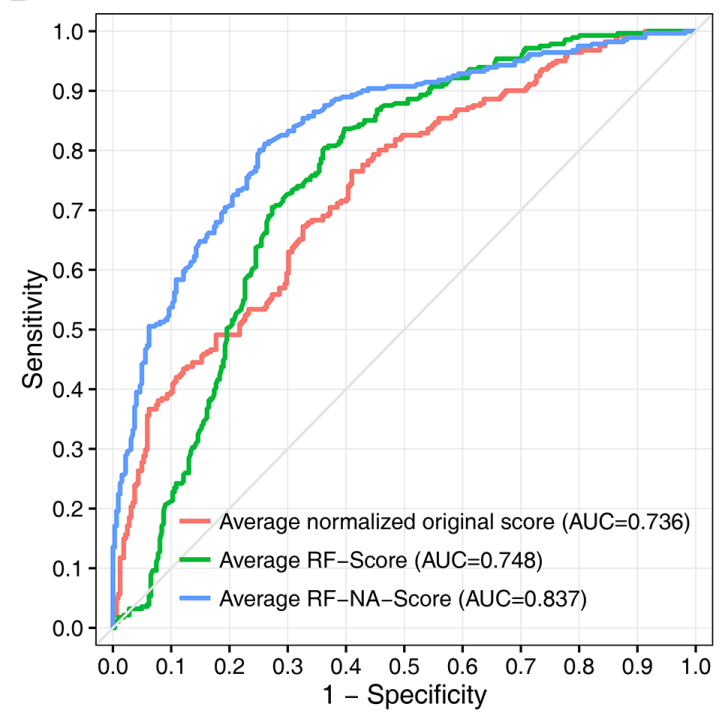

Figure 3: ROC curves for the virtual screening strategies using the docking software tools AutoDock (A), AutoDock Vina (B), and LeDock (C) combined with different scoring methods: original score (red), RF-Score (green), and RF-NA-Score (blue). Strategy using the average scores of the three docking software tools (D). 
NA inhibitors with novel scaffolds. Because these two inhibitors were screened from a lead-like library, they can be considered lead compounds for the development of new anti-influenza drugs. Our results also confirm that our virtual screening strategy is capable of discovering new NA inhibitor scaffolds.

\section{DISCUSSION}

In this study, we developed a machine-learningbased influenza-virus-NA-specific scoring function, called RF-NA-Score, using 67 NA-ligand complexes and their experimental binding affinities obtained from the
PDBbind database as the training data. The performance of RF-NA-Score in predicting the binding affinities of ligands and NA was validated with 5-CV and LOOCV, and RF-NA-Score was more accurate than RF-Score. We also validated the accuracy of AutoDock, AutoDock Vina, and LeDock in docking ligands to influenza NA. We then evaluated various docking-rescoring virtual screening strategies using different docking software tools combined with different scoring methods, using a ligand dataset containing 281 NA inhibitors and 322 noninhibitors. The results suggest that rescoring with RFNA-Score significantly improves the efficiency of virtual screening and that the best strategy involves docking with

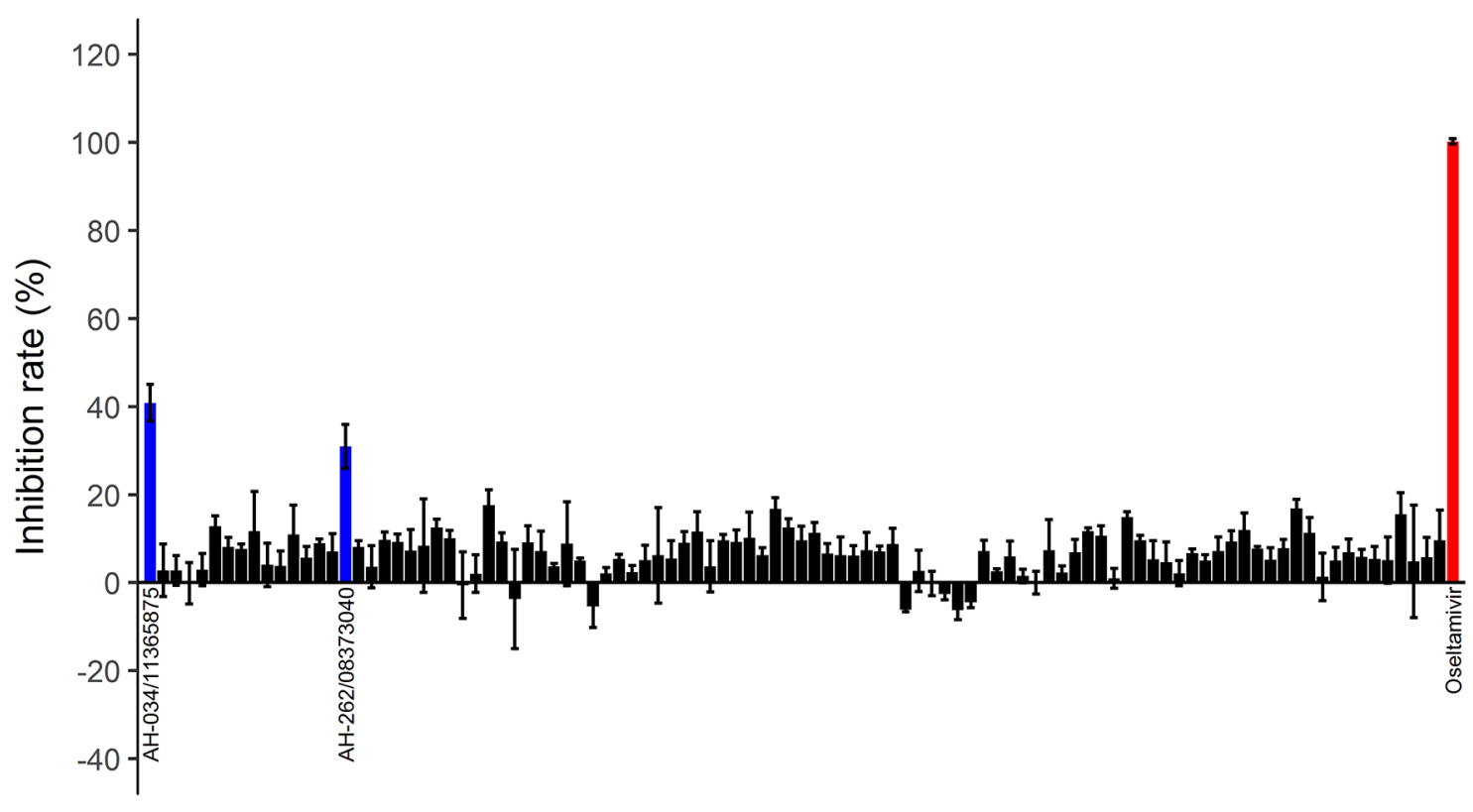

Figure 4: Inhibition rates (\%) of 100 candidate inhibitors at concentrations of $100 \boldsymbol{\mu M}$. Oseltamivir carboxylate was used as the positive control (red column). Two compounds (blue columns) inhibited NA activity by $>30 \%$.
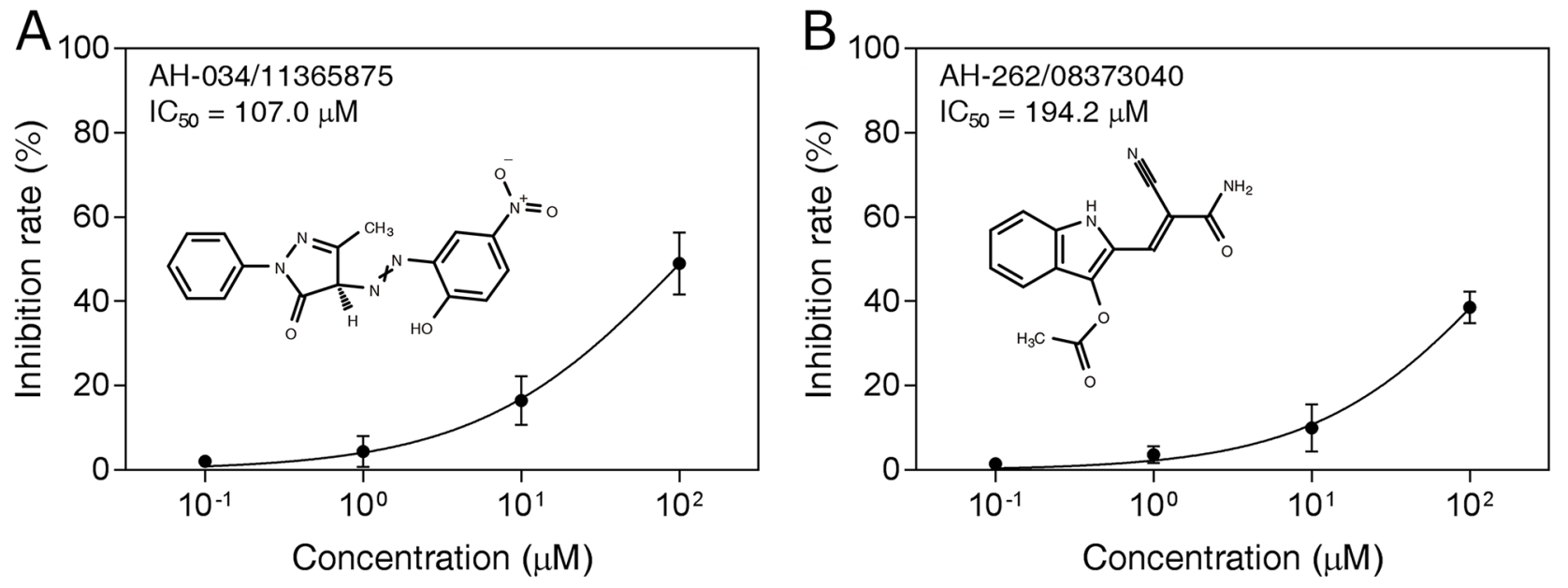

Figure 5: Dose-dependent inhibitory effects (IC50 values) of compounds AH-034/11365875 (A) and AH-262/08373040 (B). 
AutoDock, AutoDock Vina, or LeDock, rescoring with RF-NA-Score, and then averaging the scores. This strategy was then used to screen 52,631 lead-like compounds in the SPECS database. One hundred compounds were selected for testing in an in vitro H7N9 NA inhibition assay, and two compounds showed moderate anti-H7N9 NA activity. These two compounds were shown to be novel scaffolds, supporting the notable advantage of RF-NA-Score and this virtual screening strategy.

Despite the advantages of the virtual screening strategy proposed in this study, there are some noteworthy limitations. First, although we have validated the accuracy of AutoDock, AutoDock Vina, and LeDock in docking ligands to influenza NA, the docking software tools may still get incorrect results in docking other ligands, thus affecting the effectiveness of RF-NA-Score and the virtual screening strategy. Then, it needs to collect a large number of crystal structures of protein-ligand complexes as well as their experimental binding affinity values prior to the training of the machine-learning-based scoring function. But for most drug targets there is not enough data to establish a specific scoring function, which limits the scope of application of this strategy. Moreover, the proposed virtual screening strategy uses three docking software tools and a rescoring function to calculate binding affinity of a ligand, which requires significantly more computational power. If we only use the proposed strategy to evaluate the compounds that were top-ranked in traditional virtual screening, the computational cost should be significantly reduced, which should be further studied.

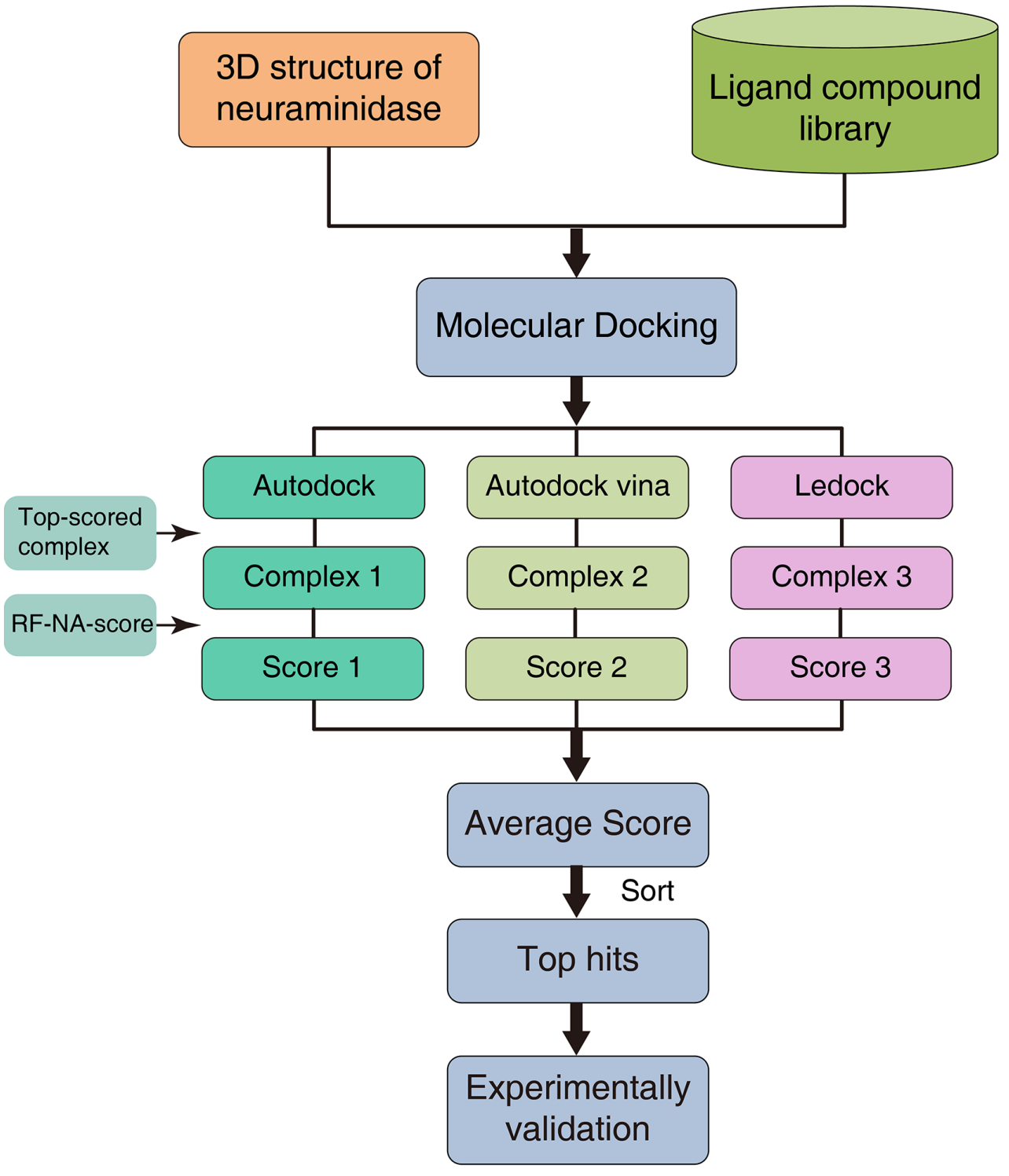

Figure 6: Virtual screening strategy using molecular docking software and RF-NA-Score. 


\section{MATERIALS AND METHODS}

\section{Data preparation}

The training dataset used to develop an influenzavirus-NA-specific scoring function for the prediction of ligand binding affinities was derived from the PDBbind database version 2016 [40], a comprehensive collection of protein-ligand complexes with high-quality crystal structures and experimentally measured binding affinity data. The binding affinity data are presented as $-\log (\mathrm{Kd} /$ $\mathrm{Ki} / \mathrm{IC}_{50}$ ) in the PDBbind database, which is a suitable format for training machine-learning-based scoring functions. In the present study, we searched the PDBbind database and collected 67 NA-ligand complexes with three-dimensional (3D) structures and binding affinity data. These 67 complexes were used as the training set for constructing the scoring function. The complete list of PDB codes and the corresponding binding affinities is provided in Supplementary Table 1. The binding affinities of the 67 selected complexes ranged widely from 2.0 to 9.74, which implies that the ligands in the training dataset had diverse chemical structures.

To evaluate the performance of the scoring functions in the virtual screening process, comprehensive searches of the literature and the bindingDB database [41] were conducted to collect small molecules with known inhibitory activities $\left(\mathrm{IC}_{50}\right.$ values) against influenza $\mathrm{A}$ virus NA. Because the aim of this study was to screen inhibitors of NA of the H7N9 influenza virus, and N9 belongs to the group 2 NAs (N2, N3, N6, N7, and N9) [42], we only collected the structures and corresponding $\mathrm{IC}_{50}$ values of group 2 NA inhibitors. In total, 706 molecules were finally collected, with $\mathrm{IC}_{50}$ values ranging from $0.1 \mathrm{nM}$ to $>7 \mathrm{mM}$. Because the molecules were collected from multiple studies reported in the literature, the methods, test conditions, and sources of NA used for the NA inhibition assays were not exactly identical [43]. Therefore, there may have been some discrepancies in the $\mathrm{IC}_{50}$ value of these molecules. Consequently, we categorized the molecules with $\mathrm{IC}_{50}<10 \mu \mathrm{M}$ as inhibitors, and those with $\mathrm{IC}_{50}>50 \mu \mathrm{M}$ as noninhibitors. The molecules in the gray area $\left(10 \mu \mathrm{M}<\mathrm{IC}_{50}<50 \mu \mathrm{M}\right)$ were removed to reduce the possible influence of variable $\mathrm{IC}_{50}$ values on the accuracy of our evaluation of the performance of the scoring functions [44-46]. Ultimately, the ligand dataset contained 281 inhibitors and 322 noninhibitors. The details of the ligand dataset are given in Supplementary Table 2.

Because there were no experimentally determined 3D structures for these collected molecules when complexed with NA, we predicted them with the molecular docking method. This is also the method used when virtual screening is performed. The accuracy of the molecular docking software tools in predicting the NA-inhibitor complexes was confirmed by testing the ability of the programs to redock the ligand into the crystal structure of NA. Four inhibitors, oseltamivir carboxylate, zanamivir, laninamivir, and peramivir, were docked to the crystal structure of H7N9 NA (PDB ID: 4MWJ), and the resulting inhibitor-NA complexes were then compared with their experimentally determined complex structures (oseltamivir carboxylate, 4MWQ; zanamivir, 4MWR; laninamivir, 4MWU; and peramivir, 4MWV).

\section{Building an NA-specific scoring function}

RF-Score is a machine-learning-based scoring function built with the random forests algorithm of Ballester and Mitchell $[33,34]$. The recent version of RF-Score has outperformed 22 state-of-the-art scoring functions on the PDBbind benchmark [35]. In the present study, we trained a new implementation of RF-Score on a refined set of the PDBbind database 2016 (containing 3766 protein-ligand complexes), based on 36 RFScore features and 11 Vina features. The NA-specific scoring function (RF-NA-Score) was trained on 67 NA-ligand complexes taken from the PDBbind database 2016 using the same method as was used to train RFScore. The performance of RF-Score in predicting the binding affinity for NA was tested on the 67 NA-ligand complexes, and the performance of RF-NA-Score was rigorously validated with 5-fold cross-validation (5$\mathrm{CV})$, leave-one-out cross-validation (LOOCV), and resampling test, which have been commonly used in the the evaluation of machine learning models [29, 47-49]. In 5-CV, the 67 complexes were randomly divided into five equal subsets: four subsets were used as training sets to predict the binding affinities of the complexes in the remaining one subset. This process was repeated five times until each subset has been used as the test set. In LOOCV, for each run, just one sample was used as the test set, and the other samples were used as the training set, and this process was repeated until all the samples has been used as the test sample. In resampling test, $70 \%$ of the complexes were randomly sampled to training data to build the scoring function, and the remaining 30\% were used to test the scoring function [50]. The test was repeated for 10 times.

The performance of the scoring functions was evaluated with RMSE, Pearson's correlation coefficient (Rp), and Spearman's rank correlation coefficient (Rs) between the predicted and measured binding affinities, because these are widely used to evaluate scoring functions [35, 51, 52]. RMSE represents the differences between the predicted and measured binding affinity values. Rp measures the linear correlation between the predicted and measured binding affinities, and Rs measures the rank correlation between the predicted and measured binding affinities. Rs shows how well the scoring function ranks the ligands according to their binding affinities. 


\section{Molecular docking calculations}

For molecular docking, the 3D structures of all the ligands used in this study were download from ZINC15 [53] or generated with CORINA Classic (https://www.mnam.com/online_demos/corina_demo). AutoDock (version 4.2.6) [54], AutoDock Vina (version 1.1.2) [55], and LeDock (version 1.0) (http://www.lephar.com/) were used for molecular docking. The crystal structure of H7N9 NA (A/Anhui/1/2013) was download from the Protein Data Bank (PDB ID: 4MWJ) and used as the receptor in the docking process. The docking site of NA was determined based on the position of the co-crystalized ligand in the structure of the NA-oseltamivir carboxylate complex (PDB ID: 4MWQ). A description of each software and how it was used in this study is given briefly below.

\section{Autodock}

AutoDock is a popular noncommercial proteinligand docking program. The scoring function of AutoDock is a semi-empirical free-energy force field. To use AutoDock, the structure files of the receptor and ligands were processed with AutoDockTools in MGLTools (version 1.5.6) [54] to merge the nonpolar hydrogen, add Gasteiger atomic charges, and set atom type to the AutoDock-supported type. During docking, the Lamarckian genetic algorithm was used to optimize the ligand conformation and 10 docking runs were performed for each ligand. The population size was set to 150 , the number of generations was set to 27,000 , and the maximum number of energy evaluations was set to $2,500,000$.

\section{Autodock vina}

AutoDock Vina is a new docking software, offering multicore capability, high performance, and enhanced accuracy [55]. AutoDock Vina uses a new knowledgebased empirical scoring function and the same receptor and ligand files as AutoDock. We used the default values for all the parameters, except that the number of CPUs was set to 1 .

\section{Ledock}

LeDock is a newly released docking program based on a combination of simulated annealing and a genetic algorithm, using a physics/knowledge-based scoring scheme. LeDock displayed high accuracy and good speed in a recent comprehensive docking program evaluation study [56]. The protein is processed by LePro (http://www. lephar.com/) and the ligands do not require processing. All the parameters were set to the default values.

\section{Virtual screening strategy using molecular docking software and RF-NA-Score}

RF-NA-Score was used for virtual screening as illustrated in Figure 6. A molecular docking software, such as AutoDock, AutoDock Vina, or LeDock, was used to predict the complex structures of NA and the ligands in the compound library. The top-scoring binding conformations for each docking software were then selected as putative complex structures. A new score for each complex structure was then calculated with a rescoring function, such as RF-Score or RF-NA-Score. An average score was also computed by averaging the scores for the complex structures predicted by the different docking software tools. The ligands were then sorted according to their scores, and the ligands with higher scores were more likely to be NA inhibitors. This process can be called a "docking-rescoring strategy".

The effectiveness of various virtual screening strategies using this docking-rescoring method, with different docking software tools combined with different rescoring functions, was evaluated based on the ligand dataset collected in this study.

The performance of the virtual screening strategies was evaluated with several methods, including score distributions, $\mathrm{p}$ values from student's $t$ test, enrichment factors, ROC curves and the areas under the ROC curves (AUCs). The score distributions of inhibitors and noninhibitors were presented as histograms and density curves. The student's $t$ test was performed to evaluate the statistical significance of the mean scores of the inhibitors and noninhibitors. Enrichment factor reflects the ability of the virtual screening method to enrich inhibitors in topscored results. In this study, the enrichment factor at $10 \%$ (EF10) was calculated using the following formula [57]:

$$
E F 10=\frac{a / n}{A / N}
$$

where $a$ is the number of actives (inhibitors) in topscored $10 \%$ compounds, $n$ is the number of compounds in $10 \%$ of the dataset (in this study $n=60$ ), $A$ is the number of actives (inhibitors) in the dataset (here $A=281$ ), $N$ is the number of compounds in the dataset (here $N=603$ ). The EF10 has an upper bound of 2.15. The ROC curves and the areas under the ROC curves (AUCs) can give more detail diagnose on performance of virtual screening method.

\section{Virtual screening of the SPECS database}

The virtual screening strategy with the best performance was used to screen for influenza virus NA inhibitors in the SPECS database (http://www.specs. net). The 3D structures of 52,631 lead-like compounds $(250<$ molecular weight $<350$, and $\log \mathrm{P}<3.5)$ in the SPECS database were downloaded from ZINC15. These compounds were subjected to the virtual screening workflow, and the 1000 top-ranking compounds were clustered by their structural similarities using the binning clustering algorithm in ChemMine tools [58], with a 
similarity cutoff of 0.4 . To increase the diversity of the candidate structures, only one compound in each cluster, with the best score, was selected for bioassay validation. Because some clusters contained many compounds, we selected slightly more compounds from these clusters. Finally, 100 candidate compounds were selected and purchased from SPECS.

\section{In vitro NA inhibition assay}

The in vitro inhibitory activities of the candidate compounds against NA were assayed with the modified method of Potier et al. [59], using oseltamivir carboxylate (MedChem Express, HY-13318) as the positive control. 2'-(4-Methylumbelliferyl)- $\alpha-\mathrm{D}-\mathrm{N}$-acetylneuraminic acid (4MU-NANA, Sigma, M8639) in MES (Sigma, M8250) buffer (32.5 mM MES, $4 \mathrm{mM} \mathrm{CaCl}$, pH 6.5) was used as the substrate and NA from A/Anhui/1/2013(H7N9) (Sino Biological Inc., 40108-VNAHC) in MES buffer was used as the enzyme. The candidate compounds were dissolved in DMSO and diluted to $500 \mu \mathrm{M}$ in MES buffer. For the assay, $10 \mu \mathrm{L}$ of the compound sample solution was mixed with $10 \mu \mathrm{L}$ of NA in a 96-well microplate and incubated for $30 \mathrm{~min}$ at $37^{\circ} \mathrm{C}$. Then $30 \mu \mathrm{L}$ of $100 \mu \mathrm{M}$ MUNANA was added and incubated for $60 \mathrm{~min}$ at $37^{\circ} \mathrm{C}$, after which $150 \mu \mathrm{L}$ of stop solution ( $0.1 \mathrm{M}$ glycine, $25 \%$ ethanol, $\mathrm{pH}$ $10.7)$ was added to each well to terminate the reaction. The fluorogenic end-product 4-methylumbelliferone (4MU) was detected with a SpectraMax M5 microplate spectrophotometer (Molecular Devices, Sunnyvale, CA, United States) at an excitation wavelength of $355 \mathrm{~nm}$ and an emission wavelength of $460 \mathrm{~nm}$. Relative fluorescence units (RFU) were calculated by subtracting the background values. The inhibition rate (IR) was calculated with the formula: IR $(\%)=\left(\mathrm{RFU}_{\text {DMSO }}-\mathrm{RFU}_{\text {sample }}\right) / \mathrm{RFU}_{\text {DMSO }} \times 100$. $\mathrm{IC}_{50}$ was calculated by plotting IR against the compound concentration, using GraphPad PRISM 7. The assays for each compound were performed in triplicate.

\section{CONFLICTS OF INTEREST}

The authors declare that they have no conflicts of interest.

\section{FUNDING}

HSL was supported by the National Natural Science Foundation of China (no. 31570160), the Innovation Team Project (no. LT2015011) of the Education Department of Liaoning Province, the Important Scientific and Technical Achievements Transformation Project (no. Z17-5-078) and the Large-scale Equipment Shared Services Project (no. F15165400) of the Science and Technology Bureau of Shenyang. HXA was supported by the Applied Basic Research Project (nos F16205151, 17231104) of the Science and Technology Bureau of Shenyang. This work was supported by the Engineering Laboratory for Molecular Simulation and Designing of Drug Molecules of Liaoning.

\section{REFERENCES}

1. Gao R, Cao B, Hu Y, Feng Z, Wang D, Hu W, Chen J, Jie Z, Qiu H, Xu K. Human infection with a novel avian-origin influenza A (H7N9) virus. N Engl J Med. 2013; 368: 188897. https://doi.org/10.1056/NEJMoa1304459.

2. Zhou L, Ren R, Yang L, Bao C, Wu J, Wang D, Li C, Xiang N, Wang Y, Li D. Sudden increase in human infection with avian influenza A (H7N9) virus in China, SeptemberDecember 2016. Western Pac Surveill Response J. 2017; 8: 6. https://doi.org/10.5365/WPSAR.2017.8.1.001.

3. World Health Organization. WHO monthly risk assessment at the Human-Animal Interface. http://www.who.int/ influenza/human_animal_interface/HAI_Risk_Assessment/ en/ (accessed date June 27, 2017).

4. Watanabe T, Watanabe S, Maher EA, Neumann G, Kawaoka Y. Pandemic potential of avian influenza A (H7N9) viruses. Trends Microbiol. 2014; 22: 623-31. https://doi. org/10.1016/j.tim.2014.08.008.

5. Uyeki TM, Katz JM, Jernigan DB. Novel influenza A viruses and pandemic threats. Lancet. 2017; 389: 2172-4. https://doi.org/10.1016/S0140-6736(17)31274-6.

6. Hurt AC. The epidemiology and spread of drug resistant human influenza viruses. Curr Opin Virol. 2014; 8: 22-9. https://doi.org/10.1016/j.coviro.2014.04.009.

7. Matrosovich MN, Matrosovich TY, Gray T, Roberts NA, Klenk HD. Neuraminidase is important for the initiation of influenza virus infection in human airway epithelium. J Virol. 2004; 78: 12665-7. https://doi.org/10.1128/ JVI.78.22.12665-12667.2004.

8. Cohen M, Zhang XQ, Senaati HP, Chen HW, Varki NM, Schooley RT, Gagneux P. Influenza A penetrates host mucus by cleaving sialic acids with neuraminidase. Virol J. 2013; 10: 321. https://doi.org/10.1186/1743-422X-10-321.

9. Bouvier NM, Palese P. The biology of influenza viruses. Vaccine. 2008; 26: D49-53. https://doi.org/10.1016/j. vaccine.2008.07.039.

10. Colman PM. Influenza virus neuraminidase: structure, antibodies, and inhibitors. Protein Sci. 1994; 3: 1687-96. https://doi.org/10.1002/pro.5560031007.

11. Von Itzstein M. The war against influenza: discovery and development of sialidase inhibitors. Nat Rev Drug Discov. 2007; 6: 967-74. https://doi.org/10.1038/nrd2400.

12. Monto AS, Robinson DP, Herlocher ML, Hinson JM Jr, Elliott MJ, Crisp A. Zanamivir in the prevention of influenza among healthy adults: a randomized controlled trial. JAMA. 1999; 282: 31-5. https://doi.org/10.1001/ jama.282.1.31.

13. Treanor JJ, Hayden FG, Vrooman PS, Barbarash R, Bettis R, Riff D, Singh S, Kinnersley N, Ward P, Mills RG. 
Efficacy and safety of the oral neuraminidase inhibitor oseltamivir in treating acute influenza: a randomized controlled trial. JAMA. 2000; 283: 1016-24. https://doi. org/10.1001/jama.283.8.1016.

14. Barroso L, Treanor J, Gubareva L, Hayden FG. Efficacy and tolerability of the oral neuraminidase inhibitor peramivir in experimental human influenza: randomized, controlled trials for prophylaxis and treatment. Antivir Ther. 2005; 10: 901-10.

15. Sugaya N, Ohashi Y. Long-acting neuraminidase inhibitor laninamivir octanoate (CS-8958) versus oseltamivir as treatment for children with influenza virus infection. Antimicrob Agents Chemother. 2010; 54: 2575-82. https:// doi.org/10.1128/AAC.01755-09.

16. Bloom JD, Gong LI, Baltimore D. Permissive secondary mutations enable the evolution of influenza oseltamivir resistance. Science. 2010; 328: 1272-5. https://doi.org/10.1126/ science. 1187816 .

17. Cass LM, Efthymiopoulos C, Bye A. Pharmacokinetics of zanamivir after intravenous, oral, inhaled or intranasal administration to healthy volunteers. Clin Pharmacokinet. 1999; 36: 1-11. https://doi. org/10.2165/00003088-199936001-00001.

18. Lionta E, Spyrou G, Vassilatis DK, Cournia Z. Structure-based virtual screening for drug discovery: principles, applications and recent advances. Curr Top Med Chem. 2014; 14: 1923-38. https://doi.org/10.2174/1568026614666140929124445.

19. Ai H, Zhang L, Chang AK, Wei H, Che Y, Liu H. Virtual screening of potential inhibitors from TCM for the CPSF30 binding site on the NS1A protein of influenza A virus. J Mol Model. 2014; 20: 2142. https://doi.org/10.1007/ s00894-014-2142-7.

20. Ikram NK, Durrant JD, Muchtaridi M, Zalaludin AS, Purwitasari N, Mohamed N, Rahim AS, Lam CK, Normi YM, Rahman NA. A virtual screening approach for identifying plants with anti H5N1 neuraminidase activity. J Chem Inf Model. 2015; 55: 308-16. https://doi.org/10.1021/ci500405g.

21. Cheng LS, Amaro RE, Xu D, Li WW, Arzberger PW, McCammon JA. Ensemble-based virtual screening reveals potential novel antiviral compounds for avian influenza neuraminidase. J Med Chem. 2008; 51: 3878-94. https:// doi.org/10.1021/jm8001197.

22. An J, Lee DC, Law AH, Yang CL, Poon LL, Lau AS, Jones SJ. A novel small-molecule inhibitor of the avian influenza H5N1 virus determined through computational screening against the neuraminidase. J Med Chem. 2009; 52: $2667-$ 72. https://doi.org/10.1021/jm800455g.

23. Cheng T, Li X, Li Y, Liu Z, Wang R. Comparative assessment of scoring functions on a diverse test set. J Chem Inf Model. 2009; 49: 1079-93. https://doi.org/10.1021/ ci9000053.

24. Waszkowycz B, Clark DE, Gancia E. Outstanding challenges in protein-ligand docking and structure-based virtual screening. Wiley Interdiscip Rev Comput Mol Sci. 2011; 1: 229-59. https://doi.org/10.1002/wcms.18.
25. Wang L, You Z, Chen X, Yan X, Liu G, Zhang W. RFDT: a Rotation Forest-based predictor for predicting drug-target interactions using drug structure and protein sequence information. Curr Protein Pept Sci. 2016. https://doi.org/10 $.2174 / 1389203718666161114111656$.

26. Huang Y, You Z, Chen X. A systematic prediction of drugtarget interactions using molecular fingerprints and protein sequences. Curr Protein Pept Sci. 2016. https://doi.org/10.2 174/1389203718666161122103057.

27. Chen X, Ren B, Chen M, Wang Q, Zhang L, Yan G. NLLSS: predicting synergistic drug combinations based on semi-supervised learning. PLoS Comput Biol. 2016; 12: e1004975. https://doi.org/10.1371/journal.pcbi.1004975.

28. Chen X, Ren B, Chen M, Liu M, Ren W, Wang Q, Zhang L, Yan G. ASDCD: antifungal synergistic drug combination database. PLoS One. 2014; 9: e86499. https://doi. org/10.1371/journal.pone.0086499.

29. Zhang L, Ai H, Chen W, Yin Z, Hu H, Zhu J, Zhao J, Zhao Q, Liu H. CarcinoPred-EL: novel models for predicting the carcinogenicity of chemicals using molecular fingerprints and ensemble learning methods. Sci Rep. 2017; 7: 2118. https://doi.org/10.1038/s41598-017-02365-0.

30. Ain QU, Aleksandrova A, Roessler FD, Ballester PJ. Machine-learning scoring functions to improve structurebased binding affinity prediction and virtual screening. Wiley Interdiscip Rev Comput Mol Sci. 2015; 5: 405-24. https://doi.org/10.1002/wcms.1225.

31. Khamis MA, Gomaa W. Comparative assessment of machine-learning scoring functions on PDBbind 2013. Eng Appl Artif Intell. 2015; 45: 136-51. https://doi. org/10.1016/j.engappai.2015.06.021.

32. Ashtawy HM, Mahapatra NR. A comparative assessment of predictive accuracies of conventional and machine learning scoring functions for protein-ligand binding affinity prediction. IEEE/ACM Trans Comput Biol Bioinform. 2015; 12: 335-47. https://doi.org/10.1109/ TCBB.2014.2351824.

33. Ballester PJ, Mitchell JB. A machine learning approach to predicting protein-ligand binding affinity with applications to molecular docking. Bioinformatics. 2010; 26: 1169-75. https://doi.org/10.1093/bioinformatics/btq112.

34. Ballester PJ, Schreyer A, Blundell TL. Does a more precise chemical description of protein-ligand complexes lead to more accurate prediction of binding affinity? J Chem Inf Model. 2014; 54: 944-55. https://doi.org/10.1021/ci500091r.

35. Li $\mathrm{H}$, Leung $\mathrm{KS}$, Wong $\mathrm{MH}$, Ballester PJ. Improving AutoDock Vina using random forest: the growing accuracy of binding affinity prediction by the effective exploitation of larger data sets. Mol Inform. 2015; 34: 115-26. https://doi. org/10.1002/minf.201400132.

36. Triballeau N, Acher F, Brabet I, Pin JP, Bertrand HO. Virtual screening workflow development guided by the "receiver operating characteristic" curve approach. Application to high-throughput docking on metabotropic glutamate 
receptor subtype 4. J Med Chem. 2005; 48: 2534-47. https:// doi.org/10.1021/jm049092j.

37. Zhao W, Hevener KE, White SW, Lee RE, Boyett JM. A statistical framework to evaluate virtual screening. BMC Bioinformatics. 2009; 10: 225. https://doi. org/10.1186/1471-2105-10-225.

38. Carhart RE, Smith DH, Venkataraghavan R. Atom pairs as molecular features in structure-activity studies: definition and applications. J Chem Inf Comput Sci. 1985; 25: 64-73.

39. Chen X, Reynolds CH. Performance of similarity measures in 2D fragment-based similarity searching: comparison of structural descriptors and similarity coefficients. J Chem Inf Comput Sci. 2002; 42: 1407-14. https://doi.org/10.1021/ ci025531g.

40. Liu Z, Su M, Han L, Liu J, Yang Q, Li Y, Wang R. Forging the basis for developing protein-ligand interaction scoring functions. Acc Chem Res. 2017; 50: 302-9. https://doi. org/10.1021/acs.accounts.6b00491.

41. Gilson MK, Liu T, Baitaluk M, Nicola G, Hwang L, Chong J. BindingDB in 2015: a public database for medicinal chemistry, computational chemistry and systems pharmacology. Nucleic Acids Res. 2016; 44: D1045-53. https://doi.org/10.1093/nar/gkv1072.

42. Russell RJ, Haire LF, Stevens DJ, Collins PJ, Lin YP, Blackburn GM, Hay AJ, Gamblin SJ, Skehel JJ. The structure of $\mathrm{H} 5 \mathrm{~N} 1$ avian influenza neuraminidase suggests new opportunities for drug design. Nature. 2006; 443: 45-9. https://doi.org/10.1038/nature05114.

43. Liew CY, Ma XH, Liu X, Yap CW. SVM model for virtual screening of Lck inhibitors. J Chem Inf Model. 2009; 49: 877-85. https://doi.org/10.1021/ci800387z.

44. Klepsch F, Vasanthanathan P, Ecker GF. Ligand and structure-based classification models for prediction of P-glycoprotein inhibitors. J Chem Inf Model. 2014; 54: 218-29. https://doi.org/10.1021/ci400289j.

45. Li B, Cong Y, Yang X, Xue Y, Chen Y. In silico prediction of spleen tyrosine kinase inhibitors using machine learning approaches and an optimized molecular descriptor subset generated by recursive feature elimination method. Comput Biol Med. 2013; 43: 395-404. https://doi.org/10.1016/j. compbiomed.2013.01.015.

46. Lv W, Xue Y. Prediction of acetylcholinesterase inhibitors and characterization of correlative molecular descriptors by machine learning methods. Eur J Med Chem. 2010; 45: 1167-72. https://doi.org/10.1016/j.ejmech.2009.12.038.

47. $\mathrm{Hu} \mathrm{H}$, Zhu C, Ai H, Zhang L, Zhao J, Zhao Q, Liu H. LPI-ETSLP: IncRNA-protein interaction prediction using eigenvalue transformation-based semi-supervised link prediction. Mol Biosyst. 2017; 13: 1781-7. https://doi. org/10.1039/C7MB00290D.

48. Chen X, Yan CC, Zhang X, You ZH, Huang YA, Yan GY. HGIMDA: heterogeneous graph inference for
miRNA-disease association prediction. Oncotarget. 2016; 7: 65257-69. https://doi.org/10.18632/oncotarget.11251.

49. Chen X, Yan CC, Zhang X, Zhang X, Dai F, Yin J, Zhang Y. Drug-target interaction prediction: databases, web servers and computational models. Brief Bioinform. 2015; 17: 696712. https://doi.org/10.1093/bib/bbv066.

50. Li J, Lenferink AE, Deng Y, Collins C, Cui Q, Purisima EO, O'Connor-McCourt MD, Wang E. Identification of high-quality cancer prognostic markers and metastasis network modules. Nat Commun. 2010; 1: 34. https://doi. org/10.1038/ncomms1033.

51. Ashtawy HM, Mahapatra NR. BgN-Score and BsN-Score: bagging and boosting based ensemble neural networks scoring functions for accurate binding affinity prediction of protein-ligand complexes. BMC Bioinformatics. 2015; 16: S8. https://doi.org/10.1186/1471-2105-16-S4-S8.

52. Zilian D, Sotriffer CA. SFCscore RF: a random forest-based scoring function for improved affinity prediction of proteinligand complexes. J Chem Inf Model. 2013; 53: 1923-33. https://doi.org/10.1021/ci400120b.

53. Sterling T, Irwin JJ. ZINC 15-ligand discovery for everyone. J Chem Inf Model. 2015; 55: 2324-37. https:// doi.org/10.1021/acs.jcim.5b00559.

54. Morris GM, Huey R, Lindstrom W, Sanner MF, Belew RK, Goodsell DS, Olson AJ. AutoDock4 and AutoDockTools4: automated docking with selective receptor flexibility. J Comput Chem. 2009; 30: 2785-91. https://doi.org/10.1002/ jcc. 21256 .

55. Trott O, Olson AJ. AutoDock Vina: improving the speed and accuracy of docking with a new scoring function, efficient optimization, and multithreading. J Comput Chem. 2010; 31: 455-61. https://doi.org/10.1002/jcc.21334.

56. Wang Z, Sun H, Yao X, Li D, Xu L, Li Y, Tian S, Hou $\mathrm{T}$. Comprehensive evaluation of ten docking programs on a diverse set of protein-ligand complexes: the prediction accuracy of sampling power and scoring power. Phys Chem Chem Phys. 2016; 18: 12964-75. https://doi.org/10.1039/ c6cp01555g.

57. Ericksen SS, Wu H, Zhang H, Michael LA, Newton MA, Hoffmann FM, Wildman SA. Machine learning consensus scoring improves performance across targets in structurebased virtual screening. J Chem Inf Model. 2017; 57: 157990. https://doi.org/10.1021/acs.jcim.7b00153.

58. Backman TW, Cao Y, Girke T. ChemMine tools: an online service for analyzing and clustering small molecules. Nucleic Acids Res. 2011; 39: W486-91. https://doi. org/10.1093/nar/gkr320.

59. Potier M, Mameli L, Belisle M, Dallaire L, Melancon S. Fluorometric assay of neuraminidase with a sodium (4-methylumbelliferyl- $\alpha$-DN-acetylneuraminate) substrate. Anal Biochem. 1979; 94: 287-96. 